\title{
An olfactory analogy to release from mixture suppression in taste
}

\author{
HARRY T. LAWLESS \\ S. C. Johnson \& Son, Racine, Wisconsin
}

In perceptually analyzable two-component odor mixtures, the perceived intensity of each component is reduced, relative to its intensity in equally concentrated unmixed stimuli. This is an example of odor counteraction, or masking. When the intensity of one component is reduced through adaptation, several sources of evidence suggest that the other component should be released from masking and increase in perceived intensity. Investigation of two-component mixtures of vanillin and cinnamaldehyde showed such a release-from-odor-masking effect. After the subjects' adaptation to vanillin, the cinnamon component of the mixture increased in perceived intensity, relative to its partially masked intensity in the mixture. A similar increase was observed for vanillin in the mixture, after the subjects' adaptation to cinnamaldehyde. This effect is consistent with a central physiological mechanism for odor masking.

In both taste mixtures and odor mixtures, mutual partial masking among components is commonly observed (reviewed in Lawless, 1986). For example, a mixture of quinine and sucrose is judged to be both less sweet than an equimolar solution of sucrose alone and less bitter than an equimolar solution of quinine alone. This effect is called mixture suppression in taste (Bartoshuk, 1975), and masking or odor counteraction in olfaction (Cain \& Drexler, 1974).

In taste mixtures, the inhibitory effect of one component on another can be undone by selective adaptation. When adaptation to one component is achieved (i.e., that component is reduced in perceived intensity), the other component of the mixture is perceptually increased in intensity. If the adaptation to one component is relatively complete, the other component is perceived at the same level as it is when unmixed, as if only a single stimulus and not a mixture were being presented (Lawless, 1979). This effect is commonly referred to as "release from mixture suppression." Various authors have argued that the effect implies a central physiological process underlying taste mixture suppression (Kroeze, 1978, 1979; Lawless, 1979).

Citing a 19th century report of Nagel (1897, in Pfaffman, 1951) and considering some of his own data on similarity judgments of odor mixtures to their components, Engen (1982, p. 119) predicted that an analogous effect should occur in olfaction. He stated that when one component of a mixture is reduced in intensity through adaptation, the other should increase in intensity, that is, it should be released from odor masking. Anecdotal evidence of such an effect exists. A well-known perfumer's trick is that, when trying to perceptually analyze a difficult complex fragrance, one should partially adapt the nose

The author thanks Kathleen Gunning for technical assistance and David A. Stevens for thoughtful discussion. Address correspondence to: H. T. Lawless, S. C. Johnson \& Son, Inc., 1525 Howe St., Racine, WI 53403. to known components. This apparently makes the other components more readily recognizable. Release from odor masking could be the basis for this practical technique. Whether such an effect occurs in olfaction was the basis of this study.

\section{METHOD}

Subjects

Eleven male subjects (mean age $=28.6$ ) and 21 female subjects (mean age $=24.0$ ) were paid for participation. Two subjects were unable to differentiate reliably unmixed cinnamaldehyde from vanillin and were excluded from the analysis.

\section{Stimuli}

Odorants were $10 \%(\mathrm{v} / \mathrm{v})$ vanillin and $3 \%(\mathrm{v} / \mathrm{v})$ cinnamaldehyde. Both were diluted in a solvent, special odorless diethyl phthalate. Stimuli were presented in 120-ml glass jars with caps. Odorants were placed in the jars by dipping perfumer's blotter strips (Red Line, Frank Orlandi, Inc.) in the odorants or solvent to a depth of $4.5 \mathrm{~cm}$, placing the strips in the jars, and capping the jars, allowing the headspace to equilibrate. Care was taken to ensure that in jars with two blotters, the blotters did not touch, to prevent physical interaction of the chemicals in the liquid phase.

\section{Design}

Jars were always presented in pairs. The first jar provided an adaptation treatment. It contained a blotter strip dipped in either vanillin, cinnamaldehyde, or solvent only. The second jar provided the stimulus to be rated. It contained a pair of blotters, either solvent and vanillin (vanillin stimulus), solvent and cinnamaldehyde (cinnamaldehyde stimulus), or vanillin and cinnamaldehyde (mixture stimulus). The strips dipped in solvent alone were included to make all three types of stimulus jars visually identical.

Nine different trials were obtained by the combinations of the three types of adapting jars with the three types of stimulus jars.

\section{Procedure}

Informed consent was obtained. The subjects were instructed in the method of magnitude estimation and given examples of each (unmixed) odorant. In each subsequent trial, the subjects gave three slow deep sniffs of the adapting jar (each lasting about $3 \mathrm{sec}$ ) and then, without inhaling room air, sniffed the stimulus jar and made their evaluation. The subjects rated the vanilla and cinnamon intensity of each stimulus. An "other" category was also provided for ratings of any odor qualities, other than vanilla and cinnamon, that the subjects might feel were present. 
Ratings were made relative to a standard ( $10 \%$ vanillin, presented before the first trial), which was designated a 10 . The nine possible adapting-stimulus pairs were presented in a different random order for each subject. A replicate session was run at least 25 min following the first session.

\section{RESULTS}

Mean ratings for each adapting-stimulus pair are shown in Table 1. Cinnamon and vanilla ratings were analyzed by repeated measures analysis of variance with adapting, stimulus, and replicate conditions as factors. Significant interactions of adapting condition $\times$ stimulus were observed for vanilla ratings $[F(4,116)=11.50, p<.001]$ and cinnamon ratings $[F(4,116)=13.86, p<.001]$. No effect of replicates or any other interaction reached significance. All statistical comparisons of individual cells discussed below were confirmed with nonparametric tests.

\section{Odor Counteraction}

Ratings of vanilla in the mixture (after adaptation to the solvent) were lower than when vanillin was presented alone, with respective means of 3.3 and $7.1[t(59)=4.36$, $p<.05$ ]. Cinnamon ratings were similarly lower for the mixture than for the cinnamaldehyde presented alone, with respective means of 11.8 and $14.3[t(59)=1.33, p<$ $.10]$.

\section{Adaptation}

Vanilla ratings were lower after presentation of the jar containing vanillin than the jar containing the solvent, with means of 4.1 and $7.1[t(59)=3.72, p<.001]$. Similarly, cinnamon ratings were lower for the cinnamaldehyde stimulus following presentation of the adapting jar containing cinnamaldehyde than after the jar containing only solvent, with means of 7.9 and $14.3[t(59)=4.62$, $p<.01]$.

\section{Release from Counteraction}

After presentation of the adapting jar containing cinnamaldehyde, the mean vanilla rating of the mixture increased from 3.3 to $7.3[t(59)=5.07, p<.001]$, which was not significantly different from its baseline rating of

Table 1

Mean Intensity Judgments

\begin{tabular}{|c|c|c|c|c|c|c|}
\hline \multirow[b]{3}{*}{ Stimulus } & \multicolumn{6}{|c|}{ Adapting Condition } \\
\hline & \multicolumn{2}{|c|}{ Solvent } & \multicolumn{2}{|c|}{ Vanillin } & \multicolumn{2}{|c|}{ Cinnamaldehyde } \\
\hline & Mean & $S E$ & Mean & $S E$ & Mean & $S E$ \\
\hline \multicolumn{7}{|l|}{ Vanillin } \\
\hline Vanilla Component & 7.1 & 0.7 & 4.1 & 0.7 & 6.9 & 0.7 \\
\hline Cinnamon Component & 0.4 & 0.1 & 0.2 & 0.1 & 4.2 & 1.1 \\
\hline \multicolumn{7}{|l|}{ Cinnamaldehyde } \\
\hline Vanilla Component & 1.3 & 0.5 & 2.4 & 0.6 & 1.2 & 0.3 \\
\hline Cinnamon Component & 14.3 & 1.9 & 14.8 & 1.8 & 7.9 & 1.0 \\
\hline \multicolumn{7}{|l|}{ Mixture } \\
\hline Vanilla Component & 3.3 & 0.6 & 3.4 & 0.7 & 7.3 & 0.7 \\
\hline Cinnamon Component & 11.8 & 1.3 & 13.6 & 1.5 & 5.9 & 0.8 \\
\hline
\end{tabular}

7.1. After presentation of the vanillin adapting jar, the mean cinnamon rating of the mixture increased from 11.8 to 13.6. However, this did not achieve statistical significance $[t(59)=1.18, p=.12]$.

\section{Other Effects}

There was no cross-potentiation of vanillin alone after adaptation to cinnamaldehyde. That is, vanillin after cinnamaldehyde was not rated as more intense than vanillin after solvent $[t(59)=0.15]$. Similarly, the cinnamaldehyde stimulus was not potentiated by adaptation to vanillin $[t(59)=0.36]$. After adaptation to cinnamaldehyde, there was a persistent rating of cinnamon for the vanillin stimulus $[t(59)=3.40, p<.001]$.

\section{DISCUSSION}

As Engen (1982) predicted, the intensity of one component of an odor mixture increases when the other component is adapted. This effect may be one reason for the perfumer's trick of selectively fatiguing the nose to a known component of a fragrance, in order to better discern the identity of other hard-to-analyze components. This practical technique may work not only because the adapted components are less pronounced, but also because the other components are released from some inhibitory process or masking.

Such sequential interactions in both olfaction and taste may explain many flavor incompatibilities. For example, dry table wines are usually poorly suited to consumption with sweet desserts. This is because the sweet desserts selectively adapt any sweet sensations that would arise from the residual sugar in the wine. This releases the sensations from the acid of the wine from suppression, making the wine appear too sour.

The release from masking following adaptation is consistent with central physiological mechanisms underlying masking. A central mechanism was previously suggested by masking in mixtures in which components are presented to separate nostrils (dichorhinically) (Cain, 1975). The release effect cannot be explained by any direct sequential crosspotentiation of one component by another. There was no increase in the vanilla rating of (unmixed) vanillin following cinnamaldehyde, relative to its baseline rating following solvent. Similarly, there was no increase in the cinnamon rating of (unmixed) cinnamaldehyde following adaptation to vanillin.

Data of individual subjects were highly variable, and not every subject showed every effect (adaptation, masking, release). The analytical task of breaking down the odor mixture into its components was difficult for many subjects. This was illustrated by the persistence of cinnamon odor in the cinnamaldehyde-vanillin sequence. Apparently, many subjects could not discern that the cinnamaldehyde had "stopped" and that only vanillin was present in the second jar. Recently, Gregson (1986) suggested that odors have both a qualitatively recognizable aspect and an aqualitative component, similar to the sensation experienced with weak stimuli between the detection and recognition thresholds. A second source of error could arise from difficulty in assigning this aqualitative component to one or to the other of the odor response categories on the ballot. Finally, previous experiments in release from mixture suppression in taste have achieved relatively complete conditions of adaptation, virtually eliminating any sensation of the adapted component. As the present conditions of adaptation were less than complete, one might expect the release effect also to be less robust. More complete conditions of adaptation might produce more complete release-frommasking effects.

Recently, O'Mahony and colleagues have demonstrated the effects of adaptation on discrimination performance in sensory product tests commonly used in industry (O'Mahony \& Goldstein, 1986; O'Mahony \& Odbert, 1985). Warm-up trials, in which the subject is preexposed to the two stimuli to be differentiated, were shown to enhance performance in a variation of three-alternative forced-choice procedures ( $O^{\prime}$ Mahony \& 
Goldstein, 1986). Enhanced performance with a warm-up procedure may in part be due to adaptation of those flavor characteristics common to the two stimuli, thus enhancing the detectability of those sensory characteristics for which the two stimuli differ, through release from masking.

\section{REFERENCES}

BartoshuK, L. M. (1975). Taste mixtures: Is mixture suppression related to compression? Physiology \& Behavior, 14, 643-649.

CAIN, W. S. (1975). Odor intensity: Mixtures and masking. Chemical Senses \& Flavour, 1, 339-352.

Cain, W. S., \& DreXler, M. (1974). Scope and evaluation of odor counteraction and masking. Annals of the New York Academy of Sciences, 237, 427-439.

ENGEN, T. (1982). The perception of odors. New York: Academic Press.

GREGSON, R. A. M. (1986). Qualitative and aqualitative intensity components of odour mixtures. Chemical Senses, 11, 455-470.

Kroeze, J. H. A. (1978). The taste of sodium chloride: Masking and adaptation. Chemical Senses \& Flavour, 3, 443-449.
Kroeze, J. H. A. (1979). Masking and adaptation of sugar sweetness intensity. Physiology \& Behavior, 22, 347-351.

LAWLESS, H. T. (1979). Evidence for neural inhibition in bittersweet taste mixtures. Journal of Comparative \& Physiological Psychology, 93, 538-547.

LAWLESS, H. T. (1986). Sensory interactions in mixtures. Journal of Sensory Studies, 1, 259-275.

O'MAHONY, M., \& GoldSTEIN, L. R. (1986). Effectiveness of sensory difference tests: Sequential sensitivity analysis for liquid food stimuli. Journal of Food Science, 51, 1550-1553.

O'MAhony, M., \& ODBert, N. (1985). A comparison of sensory difference testing procedures: Sequential sensitivity analysis and aspects of taste adaptation. Journal of Food Science, 50, 1055-1058.

Pfaffman, C. (1951). Taste and smell. In S. S. Stevens (Ed.), Handbook of experimental psychology. New York: Wiley.

(Manuscript received for publication February 9, 1987.) 